\title{
PET/MRI: The Blended-Modality Choice of the Future?*
}

\author{
Norman E. Bolus ${ }^{1}$, Remo George ${ }^{1}$, Johrnee' Washington ${ }^{2}$, and Bradley R. Newcomer ${ }^{1}$ \\ ${ }^{I}$ Nuclear Medicine Technology Program, Department of Clinical and Diagnostic Sciences, School of Health Professions, \\ University of Alabama at Birmingham, Birmingham, Alabama; and ${ }^{2}$ Montgomery Cardiovascular Associates, Montgomery, Alabama
}

\begin{abstract}
This article addresses the emerging technology of PET coupled with MRI, or PET/MRI, which could become the technology of choice in the future for many reasons. Some of these reasons will be discussed, along with a historical account of the field of MRI and how this modality has evolved to include many aspects of molecular and functional imaging. After reading this article, nuclear medicine technologists should be able to provide an overview of the history of MRI, discuss PET and how it is mainly used today melded to CT as PET/CT, discuss how MRI is used diagnostically, explain how PET technology and MRI technology are able to function simultaneously together as PET/MRI, discuss some issues concerning who will operate these new units, and discuss the possibility that PET/MRI could be the blended technology of choice in the future.
\end{abstract}

Key Words: positron emission tomography; computed tomography; magnetic resonance imaging; PET/MR imaging

J Nucl Med Technol 2009; 37:63-71

DOI: $10.2967 /$ jnmt.108.060848

\section{$\mathbf{I}_{\mathrm{n}}$} the beginning, there was a chemical identification technology known as nuclear magnetic resonance (NMR) spectroscopy. This technology was in the form of tabletop units, which were standard issue in research organic chemistry laboratories. Essentially, researchers discovered that by putting organic chemicals in a strong magnetic field, hydrogen atoms would orient themselves along the strong magnetic field. When this magnetic field was switched off, hydrogen atoms would relax back to their equilibrium state and in the process give off a radiofrequency energy signal. The energy or spectral signatures of these signals could identify them as coming from hydrogen atoms attached to different molecules. Overall, these energy spectra were the basis of NMR spectroscopy and gave scientists the ability to detect unique fingerprints for different chemicals.

Received Dec. 4, 2008; revision accepted Apr. 6, 2009.

For correspondence or reprints contact: Norman E. Bolus, MPH, CNMT, UAB NMT Program, 1705 University Blvd., SHPB, Room 441, Birmingham, AL 35294-1212.

E-mail: bolusn@uab.edu

*NOTE: FOR CE CREDIT, YOU CAN ACCESS THIS ACTIVITY THROUGH THE SNM WEB SITE (http://www.snm.org/ce_online) THROUGH JUNE 2011. COPYRIGHT @ 2009 by the Society of Nuclear Medicine, Inc.
In the 1970s, this same spectroscopic technology was expanded into the diagnostic imaging realm. Essentially, scientists developed the ability to apply the same NMR signal as is used in spectroscopy studies to a technology that was able to scan an entire organ system. This technology was first demonstrated in organic objects such as fruits and then in animal organ systems, and then it quickly was extended to humans.

The initial technologic challenge was to build instruments large enough to scan a human yet robust enough to maintain strong and stable magnetic fields over long periods. This challenge was finally met with the advent of magnets constructed with materials able to operate in the superconductive realm (i.e., the realm in which electrical energy loss due to resistive losses is near zero). In the early to mid 1980s, when NMR first emerged as a widespread clinical imaging modality, it originally fell under the domain of nuclear medicine technology as an offshoot of nuclear medicine. Additionally, the very first NMR technologists were nuclear medicine technologists. Furthermore, some of the more progressive nuclear medicine technology educational programs taught NMR physics and provided students with opportunities to experience clinical rotations in NMR. Unfortunately, once the modality began to mature and after Medicare began to reimburse for NMR procedures, many in the field of NMR realized that the word nuclear in the title was misleading the general public to believe ionizing radiation was being used as it was in nuclear medicine. Because this was not the case for NMR, the word nuclear was dropped from the title and thus the field of magnetic resonance imaging (MRI) was born.

Eventually, MRI broke away from nuclear medicine and became a separate imaging modality. The original role of nuclear medicine technologists faded as more radiologic technologists and more technologists who had been trained on the job entered the field. Interestingly, most recently, MRI is back to using the original spectroscopy technology on clinical machines whose first application was to identify unique biochemical signatures associated with disease states or conditions within the human body.

The recent introduction of PET/MRI technology is considered by many experts to be a major breakthrough that will potentially lead to a paradigm shift in health care and 
revolutionize clinical practice (1). Since the introduction of SPECT/CT in 1998 and PET/CT in 2000, blended imaging devices have become the modality of choice for both the medical imaging and the research communities because of the unmatched levels of diagnostic information provided by these hybrids. Hybrid imaging devices complement each other by adding information that otherwise would not be available with individual stand-alone units (2). Clinical results have shown that the combination of functional information (from PET) and anatomic structures (revealed by $\mathrm{CT}$ ) into a single image provides an advanced diagnostic tool and research platform (3). Although PET/CT is already an established clinical tool, it still bears some limitations. Even though CT measurements for PET attenuation correction greatly enhance image quality and reduce PET scanning time, they also tie up the CT scanner (i.e., if the PET/CT scanner is used diagnostically for both modalities or used part of the time as solely a CT scanner) and increase the overall radiation dose to the patient (4). Also, it is argued that the anatomic data of some organ systems and tissues derived from CT is not as complete as that which could be obtained with MRI, and the metabolic information that can be obtained with PET is enhanced by that which might be obtained with MRI, especially with functional MRI (fMRI) and MR spectroscopy (MRS) (2,3). These arguments have led to the recent development of combined PET/MRI units, which are being promoted as even better than PET/CT. The main purpose of this article is to examine the advantages and drawbacks of each technology while broaching the question of whether PET/MRI could potentially carve out a niche for itself as the future modality of choice in the field of molecular imaging.

\section{PET}

PET is a nuclear medicine imaging modality that uses $\gamma$-rays produced by positrons annihilating with electrons. These positrons emitted from certain injected radionuclides or radiopharmaceuticals are used to determine the metabolic activity of living cells. The injected positron-emitting radionuclides usually have a short half-life and include ${ }^{11} \mathrm{C}$ ( $\sim 20 \mathrm{~min}),{ }^{13} \mathrm{~N}(\sim 10 \mathrm{~min}),{ }^{15} \mathrm{O}(\sim 2 \mathrm{~min})$, and ${ }^{18} \mathrm{~F}(\sim 110$ min), among others. As positrons travel through matter, they interact with electrons, resulting in an annihilation reaction that produces 2 pairs of $511-\mathrm{keV} \gamma$-rays emitted simultaneously in nearly $180^{\circ}$ opposing directions.

$$
\beta^{+}+\mathrm{e}^{-} \rightarrow \operatorname{photon}(511 \mathrm{keV})+\operatorname{photon}(511 \mathrm{keV})
$$

Inside the PET unit, hundreds of detector blocks made of scintillating materials such as bismuth germanium oxide, lutetium oxyorthosilicate, or gadolinium oxyorthosilicate are arranged in consecutive full or half rings surrounding the patient cylindrically, enabling the detection of many slices of coincidence data emerging from the patient at one time. The path between 2 detectors is referred to as a line of response (LOR). Each pair of detectors in the ring defines a possible emission path. The interval during which 2 annihilation photons must strike the detector in order to be considered in coincidence is typically $12-15$ ns. Those coincidence events that fall outside this range consist usually of undesired random and scatter data and are rejected by the coincidence timing circuitry. Some modern, fast scintillators can accurately estimate the location of an annihilation event along the LOR by measuring the difference in the arrival times of the annihilation photons at the opposing detectors (time-of-flight camera). With time-offlight information, the annihilation point can be localized to a limited range within the LOR, whereas without time-offlight information, annihilation is assumed to be located with equal probability along the entire LOR. In the latter case, many intersecting LORs are needed for the precise 3-dimensional localization of the activity, whereas in the former detector types, this information is identified both within the LOR and by the intersecting LORs.

Often, toward the edges of the field of view of the gantry, penetration of $511-\mathrm{keV}$ photons into the crystal ring blurs the measured position, because the detectors at the edge lie at an angle to some of the incident beams and have less material to stop the beam. The result is a spillover of the photon to the adjacent crystal at that angle, which may or may not stop that beam (Fig. 1). These variations in the depth of interactions that can occur for a ray within the crystals at the edge, in comparison to those toward the center, is sometimes referred to as parallax error or radial elongation and can degrade the axial resolution at the edges of the field of view. Therefore, sources of radioactivity near the edges of the gantry ring tend to have poorer resolution (wider full width at half maximum) than do sources at the center.

When the $511-\mathrm{keV}$ photons strike the scintillation crystal, they are absorbed within the crystal lattice and produce light photons that strike the photocathode of a photo-

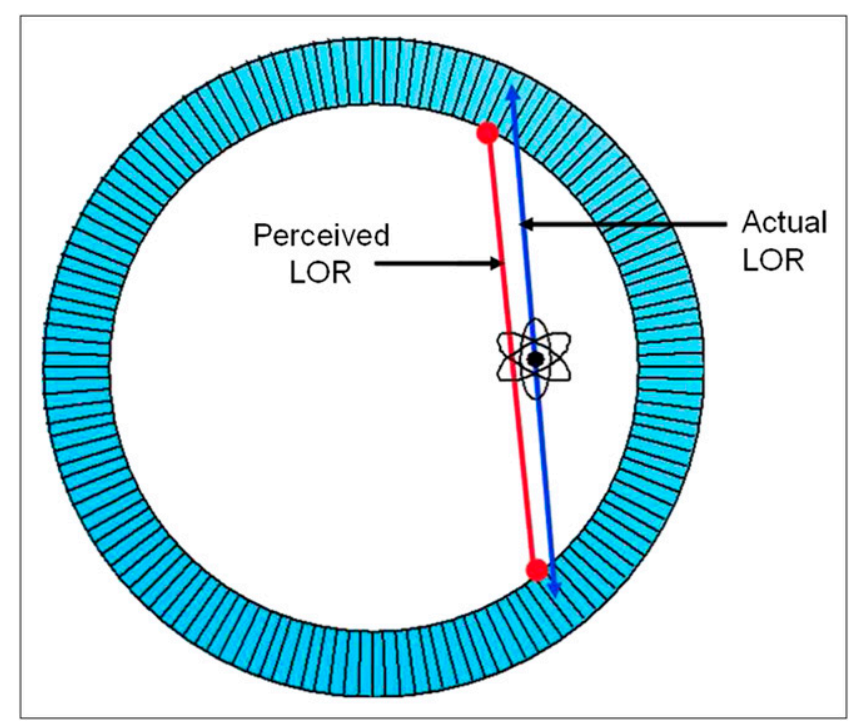

FIGURE 1. Perceived vs. actual line of response (LOR) in PET imaging. 
multiplier tube coupled to the crystal. A pulse is generated at the photomultiplier tube and then increased by an amplifier. The energy and spatial position of the signal are determined, and finally a count is recorded. When 2 such events are detected by a pair of opposing detectors simultaneously within the time window, a coincidence event is recorded. The total time required to complete these steps-from the striking of the crystal by the annihilation photon pair to its being recorded as an annihilation event-is defined as the dead time. During this time, the detection system is unable to process a second event arriving at the crystal, which will be lost. These losses (called dead-time loss) increase as the counting rate increases and can be a significant source of count loss for PET. Modern PET systems strive to minimize these count losses using faster scintillators (detectors with shorter scintillation decay times) and faster processing electronics.

Annihilation radiation may also undergo Compton scattering while passing through body tissues and can fall within the energy window of the pulse-height-analyzer setting for 511-keV photons, resulting in their undesired detection by a detector pair within the coincidence timing window. These scatter events can occur outside or inside the detector field of view. Scatter coincidences are caused when scattered photos are misdirected to false locations on the detector to form coincidence events, and random coincidences occur when 2 disintegrations occur within the timing window and 1 photon from each creates a false coincidence (Fig. 2).

These scattered radiations can increase the image background, thus degrading image contrast. The scatter contribution increases with the density and depth of the body tissue, the density of the detector material, the activity administered, and the pulse-height-analyzer window width. Even though the pulse-height window cuts off a large fraction of the scattered radiation, a significant amount of scatter is still let in by the pulse-height analyzer. Additional scatter correction is provided by the introduction of thin septa into the field of view (2-dimensional acquisition). These septa act like collimators in $\gamma$-camera systems, limiting and defining the direction and angular divergence of the incident photons (Fig. 3) and reducing interplane scatter significantly, but, unlike the collimator of a $\gamma$-camera, do not provide any additional spatial resolution.

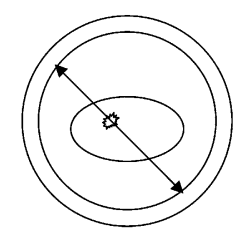

True coincidence
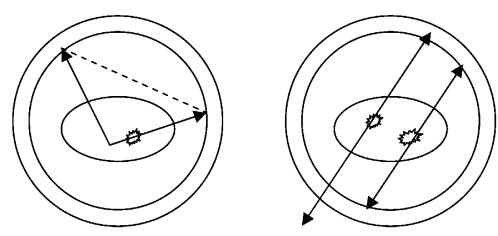

Scatter coincidence
FIGURE 2. True coincidence detection vs. scatter and random coincidence detection in PET imaging.

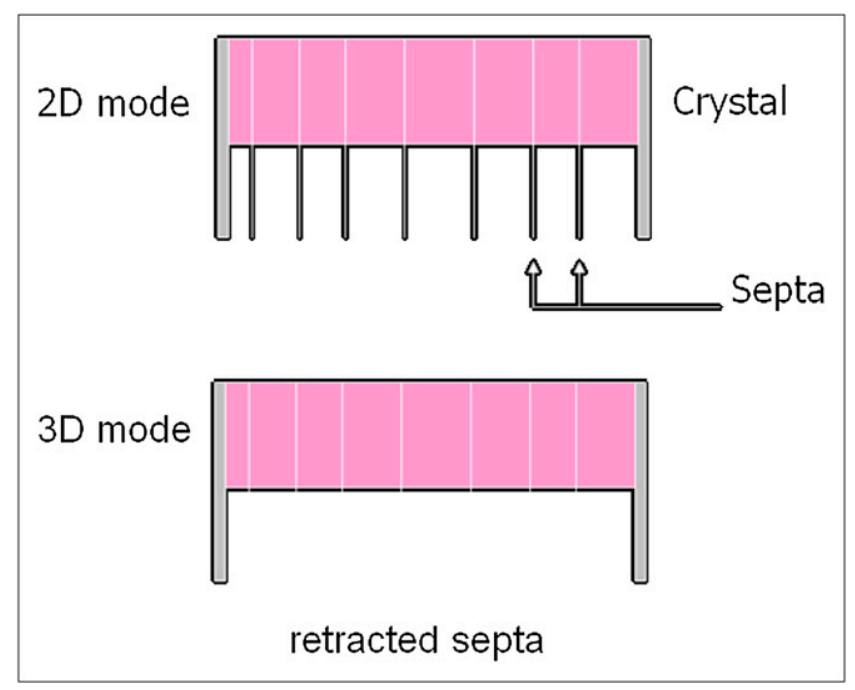

FIGURE 3. $2 \mathrm{D}$ vs. 3D PET imaging.

Two-dimensional acquisition can, however, result in lower overall counting sensitivity because of the significant number of events (scattered and some true) discarded by requiring longer acquisitions or higher injected dosages to compensate. An alternative to this impediment is to acquire counts without any septa (3-dimensional acquisition) (Fig. 3 ), an option that would increase the sensitivity significantly - the tradeoff being higher scatter and more random events detected. These disadvantages of 3-dimensional acquisitions are usually compensated using better reconstruction algorithms and rebinning techniques (data reformatted to resemble 2-dimensional data before reconstruction).

The 511-keV photons originating from different locations in the patient body are attenuated (lose energy) as they traverse different thicknesses of tissue to reach the detector pair in coincidence. Photon attenuation causes significant image nonuniformities due to the loss of coincidence events resulting from the 2 photons traversing different organs (with different attenuation coefficients) along the LOR and reaching the detectors outside the coincidence timing and pulse-height acceptance windows. Therefore, corrections must be made for this attenuation of photons in the body tissue to facilitate their inclusion as true coincidence events. In the transmission method for attenuation correction, 2 scans are obtained: a blank scan without the patient in the scanner (performed as part of daily quality assurance at the beginning of the day), and a transmission scan with the patient positioned in the scanner (transmission sources can be a set of long-lived ${ }^{68} \mathrm{Ge}$ rod sources or ${ }^{137} \mathrm{Cs}$ point sources). Correction factors are generated for each detector pair (i.e., each LOR) as in Equation 1.

$$
\mathrm{I}_{0} / \mathrm{I}=\mathrm{e}^{\Sigma \mu_{\mathrm{i}} \mathrm{D}_{\mathrm{i}}}
$$

where $\mathrm{I}_{0}$ is the blank scan data, $\mathrm{I}$ is the measured transmission scan data, and $\mu_{i}$ and $D_{i}$ are, respectively, the linear attenuation coefficient and thickness of the number (i) of organs or 
tissues through which the photon travels. These factors are then applied to correct all individual LOR counts in the patient study data. The low-resolution rod source is replaced by the high-resolution CT scan in PET/CT units.

PET noninvasively images the in vivo distribution of injected biomolecules (small molecules, peptides, antibodies, and nanoparticles) labeled with positron emitters. The high sensitivity of PET allows measurement of picomolar concentrations of labeled biomolecules. However, the spatial resolution of PET is limited by physical factors associated with positron physics (scatter, attenuation, and radial elongation) and by the difficulty of acquiring sufficient counting statistics (dead-time losses, 2-dimensional vs. 3-dimensional acquisition, and scatter correction techniques). Thus, PET images often lack definitive anatomic information, making interpretation of the precise location of radiotracer accumulation difficult (5).

\section{MRI}

MRI scanners have proven so clinically useful because the MRI signal has a greater information density than is available through any other imaging modality. MR images can use this increased information density to provide multiplanar high-spatial-resolution anatomic images with exquisite soft-tissue contrast $(6,7)$. The information content in the clinical MRI signal is based on 5 components: the density of hydrogen molecules in a given tissue, the longitudinal or spin-lattice relaxation (T1) properties of a given tissue, the transverse or spin-spin relaxation (T2) properties of a given tissue, the chemical shift properties of the hydrogen molecules in a given tissue, and motion and flow phenomena. Each of these components will be briefly discussed below.

The first information component in the MRI signal is the density of hydrogen molecules, or proton density. The proton density in a given tissue is dependent on its concentration of various hydrogen-containing molecules. Clinically, the main hydrogen-containing molecules seen in MR images are associated with water and fat molecules. Furthermore, the density of hydrogen atoms in a particular tissue can be manipulated through the administration of various MRI contrast agents, such as gadolinium-based agents. Overall, the tissue proton density is the most basic form of contrast in a clinical MR image, is a result of the differences in water and fat concentrations in a given location, and can be affected by various pathologies and contrast agent administrations.

The second and third information components in the MRI signal are the $\mathrm{T} 1$ and $\mathrm{T} 2$ relaxation properties of a given tissue location. These relaxation properties are dependent on the chemical environments of the hydrogen atoms. For instance, hydrogen atoms attached to an oxygen molecule in water are in a significantly different chemical environment than are hydrogen atoms attached to carbon atoms on the fatty acid chains of triglyceride molecules in fat. These types of differences allow clinical MR images to easily differentiate between signals coming from water and those coming from fat and produce some of the most striking contrast difference in the images. Furthermore, more subtle chemical environmental changes can change the relaxation properties of hydrogen atoms even if they are on the same molecules (i.e., water molecules will have different T1s and T2s based on the type of tissue in which they are located). For instance, hydrogen atoms in the brain, gray matter, white matter, and cerebrospinal fluid will have different $\mathrm{T} 1$ and $\mathrm{T} 2$ relaxation properties based on the different chemical environments of these tissues. Overall, clinical MR images can exaggerate these differences during signal acquisition to produce images with striking contrast differences between different tissue types and between normal and abnormal tissues.

MRI contrast agents, such as gadolinium-based agents or iron-oxide nanoparticles, can also be administered intravenously to patients to affect tissue T1s and T2s (8). Several laboratories are also developing targeted MRI contrast agents based on certain tissue metabolic properties (9-11). Basically, these contrast agents confine themselves to the blood system and will alter the local magnetic fields around the paramagnetic gadolinium or iron-oxide particles. These altered local magnetic fields will in turn change the T1s and T2s of the tissues in which the contrast agent has been deposited and will cause an extreme tissue contrast difference in the resulting images. Clinically, examples of gadoliniumbased contrast agents are used to investigate areas of the brain where the blood-brain barrier has broken and in studies that investigate joints and the spinal system for areas of damage where pooling of blood may be suspected. Overall, the development of contrast agents and the enhanced manipulation of tissue contrast will be areas of future development, especially as molecular imaging studies are established on human MRI platforms.

A final and subtle set of tissue relaxation changes can be in the effect that intrinsic oxygenated blood can have on a local tissue environment. This effect is predominantly seen in brain studies and is referred to as blood oxygen leveldependent contrast changes. These changes are caused by the iron particles in the hemoglobin of oxygenated blood. As tissue is activated, the body increases the delivery of oxygenated blood to that location. Consequently, this increased oxygenated blood will bring increased amounts of iron to that tissue location, which in turn will alter the local magnetic fields around that activated tissue location. Overall, these blood oxygen level-dependent effects produce subtle changes in tissue relaxation properties that can be used to produce images with contrast related to the level of activation. This is the basis of fMRI image acquisitions. In research environments, fMRI is used to study a wide range of brain activation questions. An interesting area of current fMRI research is a set of studies looking at brain activation patterns in obese, overweight, and normal-weight individuals as they see and smell various types of foods. 
Another interesting area of research is in the development of fMRI techniques for the detection of lying. Clinically, fMRI is predominately used in the study of epilepsy and stroke and in analyses before cancer surgery.

The fourth information component in the MRI signal is the chemical shift difference, which is based on chemical differences in molecular environments between various hydrogen-containing molecules. Chemical shift differences are the basis of the original NMR spectroscopy technology. Essentially, these differences will be seen in the MRS signals between hydrogen atoms on different chemical species and will produce different chemical signatures in the MRS signal. As an example, in the brain, hydrogen will have slightly different MRS signal properties on water, fat, $\mathrm{N}$-acetylaspartate, creatine, choline, and various other neurotransmitters. Fortunately, during a clinical imaging acquisition, these chemical shift differences are small, compared with the voxel sizes used in our images today, and we have to worry about (and correct for) only the chemical shift differences between water and fat. However, these same chemical shift differences can be used to produce MRS images that represent the chemical composition of tissue noninvasively in clinical subjects $(12,13)$. This information can also be used to acquire MRS data on large tissue volumes for a similar analysis of the chemical composition of a tissue. Overall, MRS allows the relative concentrations of certain abundant metabolites, and some drugs administered at mass levels, to be measured. Clinically, proton MRS is particularly useful in tumor studies and also offers information about neuronal integrity in neurodegenerative disorders $(14,15)$. Unfortunately, one of the biggest drawbacks to MRS is its low molar sensitivity. This sensitivity for different intrinsic metabolites and tracers can be many orders of magnitude lower than that of PET, imposing significant restrictions on the kinds of targets that can be visualized with MRS. However, even with this limitation, future developments in MRS and MRS images are on the horizon as manufacturers and researchers develop stronger MRI magnets and better acquisition techniques. These future developments should allow clinicians the ability to noninvasively assess tissue biochemistry in select circumstances without the use of tissue biopsies.

The fifth information component in the MR signal is the motion and flow phenomena seen in the acquired signals. Overall, MR image acquisitions are based on the precise timing of hardware events. This requirement for precise timing makes MRI highly sensitive to flow and motion events in tissues and subjects. Clinically, these effects were considered a nuisance and classified as artifacts (e.g., ghosting artifacts, motion artifacts, and flow artifacts). However, over the past 20 years, clinical scientists have learned how to use this information to their advantage to produce images of these flow or motion events. For instance, the MRI signal can be acquired and processed to produce images of gross blood flow through the vascular system. These are referred to as MR angiography studies, or MRAs, and are seen in many of the main vascular systems in the body. The MRI signal can also be acquired and processed to produce images of blood flow through tissue capillary beds and are called MRI perfusion studies. These perfusion studies have been used in many of the main organ systems in the body (e.g., brain, heart, muscle, and liver). Another interesting MRI acquisition method is called diffusion imaging and used the flow sensitivity of the MRI signal to image the molecular motion of water at the cellular level. Diffusion imaging has been used mainly in stroke imaging to detect areas of increased water mobility where breakdowns of the blood-brain barrier or cellular death has occurred. This continues to be an area of fascinating future developments in clinical MRI and should provide many exciting technologic developments in the coming years.

In summary, the combination of high spatial resolution and exquisite tissue contrast differentiation allows the anatomic consequences (e.g., tumor growth, brain atrophy, and cardiac wall motion abnormalities) of many disease processes to be visualized in patients and in animal models. As stated previously, MRI techniques can be used to measure important physiologic tissue properties that are related to function as well as anatomic information. These functional tissue properties include tissue diffusion, tissue permeability, and changes in blood oxygenation levels after neuronal activation (8-23). Overall, the MRI and MRS technologies should continue to prove extremely valuable in the clinical diagnosis and tracking of various disease processes. Upcoming developments such as the integration of PET with MRI technologies should only increase the clinical utility and value.

\section{PET/MRI}

Clear synergy exists between PET and MRI, because each can provide unique information not attainable with the other. For this reason, effort is under way for MRI and PET to be combined in clinical diagnostics and research. Of late, these modalities have been increasingly used in more basic biomedical research, particularly in efforts to understand the etiology and evolution of human diseases in appropriate animal models (commonly mice and rats) and in the preclinical evaluation of new therapeutic strategies, including small-molecule drugs, peptides and antibodies, cellular therapies, gene therapy, and nanoparticle-based therapies. In particular, these modalities can be used in combination to study both the pharmacokinetics and the pharmacodynamics of new therapeutics (5).

In designing an integrated scanner for simultaneous PET and MRI, an obvious challenge relates to the ways in which the PET and MRI systems can interfere with each other. This interference has historically led to major artifacts or image degradation in both technologies. The primary concerns are electromagnetic interference and the effect of the main magnetic field of the MRI scanner on the detectors in 
the PET scanner. However, other more subtle effects that need to be considered include the induction of eddy currents, susceptibility artifacts, and an increase in temperature or vibrations induced by the running of MRI sequences. The traditional PET detectors (usually based on scintillators coupled to photomultiplier tubes) and associated electronics commonly used in PET scanners are highly sensitive to magnetic fields and contain conducting and radiofrequency radiating components that have the potential to interfere with the MRI system (5). Furthermore, the static main magnetic field of MRI prevents the use of classic photomultiplier tubes for radiation detection in PET. Consequently, the design of the PET components has to be fundamentally changed in order to merge the PET and MRI modalities into a single imaging system.

Many design configurations have been proposed to circumnavigate this issue. One early method was to use fiberoptic cables to transport the scintillation light from the detector crystals inside the magnet to photomultiplier tubes that reside outside the bore of the MRI scanner. Although this method led to proof-of-principle data, including a small number of animal studies, the performance of the PET scanner was poor, compared with that of standalone PET scanners, and the length of the optical fibers made the system cumbersome (5). Another design configuration was based on the use of detector readout technologies insensitive to magnetic fields. These readout technologies included avalanche photodiodes and, more recently, silicon photomultipliers (24). Other proposed configurations include the use of novel split-magnet designs that provide space for a large number of PET detector modules, and the use of field-cycled MRI systems.

Recently, one of the major vendors married a PET insert to a 3-T MRI head scanner (25), helping push the PET/MRI technologic development into the realm of human research settings. Prototypes are being installed in major U.S. and European institutions, with the possible extension to clinical whole-body PET/MRI in the future.

Until the recent introduction of PET/MRI hybrid technology, PET and MR images were acquired on separate imaging systems and typically coregistered using software that uses the information content of the image data (e.g., landmarks) or external fiducial markers that can be clearly identified in the 2 images (26). This approach works well in the brain, where the skull constrains movement and enables simple rigid-body registration methods (27). However, the approach becomes more problematic in the thorax, abdomen, and pelvis, where tissues and organs deform on the basis of the position of the subject in the scanner, and where temporal changes such as emptying of stomach contents, movement of the food through the intestinal tract, and filling of the urinary bladder also confound registration. Deformable image registration techniques can be used, but their success is highly situation-dependent, and they are not generally robust in the presence of significant tissue movement between the 2 separate imaging studies. Simultaneous acquisition, therefore, would guarantee spatial registration of the 2 datasets (5). More crucially, sequential PET and MRI (as used in today's PET/CT systems) does not permit temporal correlation of PET and MRI studies. Biologic systems are inherently dynamic, and their response to drugs and contrast agents is strongly time-dependent. The biodistribution of most contrast agents and drugs exhibits changes of seconds to minutes. Thus, data must often be acquired simultaneously to ensure that a subject is being imaged in the same physiologic state and to correlate changes over time in the PET and MRI signals in response to an intervention (5). In contrast to PET/CT, data acquisition with new hybrid PET/MRI scanners provides the option of real simultaneous studies.

A limitation of current PET/CT technology is that data are acquired sequentially rather than simultaneously (28). Sequential scanning renders impractical an accurate temporal correlation of nonrepeatable functional in vivo processes - a major restriction of current-generation PET/CT scanners (29).

Another aspect is patient motion. Trouble arises when the patient moves either voluntarily or involuntarily between or during the CT and PET data acquisitions. Motion might take place, for instance, if the patient changes position while lying on the scanner bed. Patient motion might also be due to respiration, cardiac motion, peristalsis, or bladder filling, any of which can lead to motion blurring or misregistration between PET and CT data (30). To avoid respiratory motion-induced artifacts, one may use an adequate pulse sequence with breath holding. The mediastinal motion, if any, during simultaneous scanning will be mutual and in phase for both PET and MR images and will not affect image coregistration as much as when occurring out of phase in a serial PET/CT mismatch. In general, motion artifacts may be a larger issue in PET/CT than in PET/MRI. In small-animal studies, simultaneous scanning reduces time under anesthesia and enables scanning under identical physiologic conditions. The capability of instantaneous fusion of anatomic and functional data with PET/MRI (31-33) and simultaneous scanning will resolve many of the impediments that PET/CT currently faces in the precise coregistration of anatomomolecular information and in accurate attenuation correction.

Figure 4 shows the essential steps of a typical PET/CT scan, demonstrating the degree of integration available in a modern dual-modality imaging system.

\section{Image Acquisition Times}

The scan duration of PET/MRI is anticipated to be similar to or slightly longer than that of PET/CT, depending on the MRI pulse sequence used (31). This duration can, however, be achieved only if the design of the PET/MRI scanner allows for concurrent rather than sequential data acquisition, as is the case with PET/CT. It is anticipated that the development of concurrent scanning technology will be able to keep the scan duration with PET/MRI comparable 


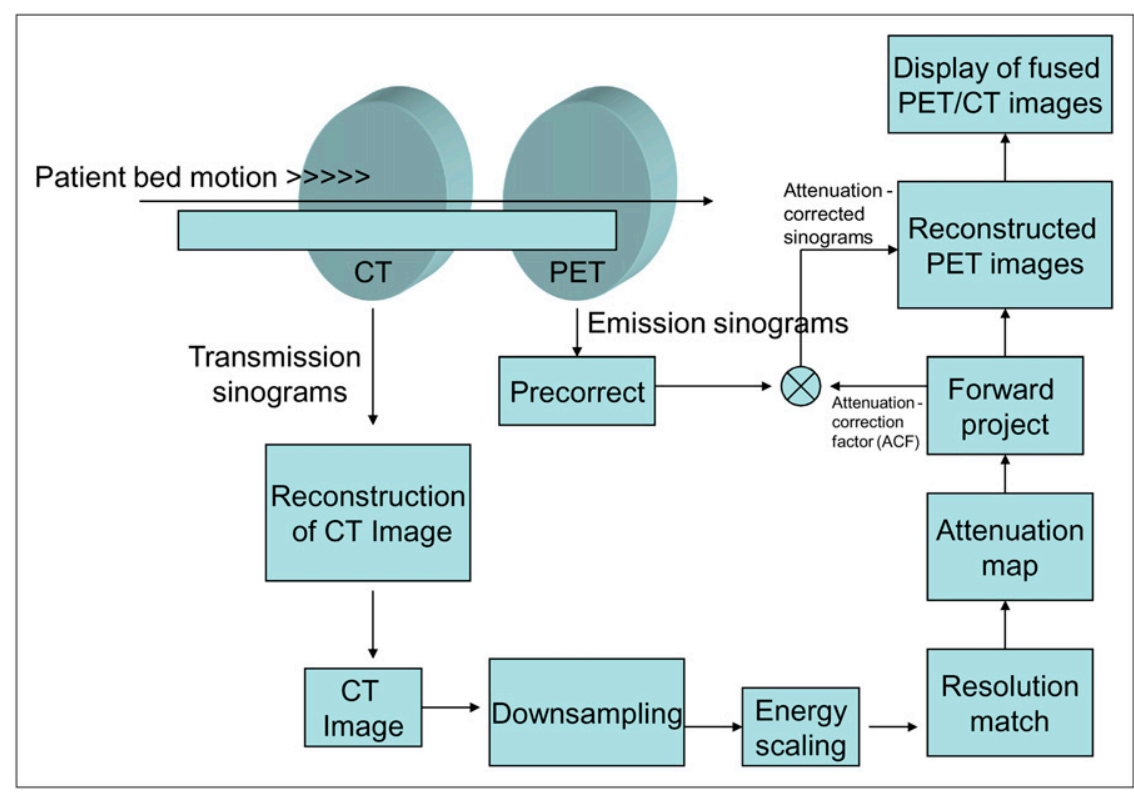

FIGURE 4. PET/CT scanner with sequential imaging. to that of sequential scanning with PET/CT. In any case, shorter scan times both improve patient comfort and reduce the time during which patient motion can occur, as well as increase patient throughput and thereby boost system use and improve cost-effectiveness.

\section{Attenuation Correction}

In comparison to $\mathrm{CT}$, MRI produces anatomic images from which it is more difficult to derive maps for attenuation correction of the PET emission data. However, some solutions do exist as demonstrated by a proof of concept for using segmented MRI-guided attenuation compensation in brain PET (34), and plenty of opportunities remain for creative advances in MRI-guided attenuation correction in whole-body PET

Use of MRI for attenuation correction, although challenging, is also presumably feasible as has been shown at least for brain imaging $(34,35)$.

\section{Absorbed Dose \\ One area in which PET/MRI has a clear advantage over PET/CT is lower exposure of patients to radiation. This advantage should, by itself, be sufficient to predict that PET/MRI could replace PET/CT. PET/CT delivers a high absorbed radiation dose, compared with the dose from a regular chest radiograph. There is some concern that multiple studies on pediatric patients could lead to an accumulated dose approaching levels (1 Sv) that can produce late, chronic effects, such as possible leukemia induction (36). Because MRI does not use any ionizing radiation, it can conceivably be used without restrictions in serial studies, in children, and in many other situations in which radiation exposure could be a concern.}

\section{Anatomic Information}

MRI yields unique morphologic and functional information unobtainable by other imaging modalities. High-field MRI generates high-resolution anatomic and structural images offering better soft-tissue contrast resolution and a large variety of tissue contrasts, compared with CT (2)

\section{Functional Information}

In addition to offering a diversity of tissue contrasts, MRI provides a wealth of additional information through fMRI and MRS to enhance the diagnostic performance and quantitative capabilities of PET and help improve patient management and the understanding of tumor biology $(37,38)$. PET/MRI allows for fMRI, thus enabling temporal correlation of blood flow with metabolism or receptor expression in brain studies and, more importantly, is capable of assessing flow, diffusion, perfusion, and cardiac motion in a single examination. MRI can be combined with MRS to measure spatially matched regional biochemical content and to assess metabolic status or the presence of neoplasia and other diseases in specific tissue regions (2). In comparison to the MRI capability of augmenting PET functional attributes, because of its low sensitivity, perfusion is the only in vivo functional information provided by $\mathrm{CT}$ in contrast-enhanced studies.

\section{Cost}

The question of how much a hybrid PET/MRI scanner will cost has yet to be answered. It is known that a dedicated PET scanner costs somewhere between $\$ 700,000$ and \$1 million. Because PET/CT scanners have been developed and have proven to be as efficient as and more diagnostically relevant than PET units alone, the purchase of dedicated PET scanners is becoming a thing of the past. The average price of a PET/CT scanner is $\$ 1.5$ 
million, but prices can go up to as high as $\$ 2.5$ million. MRI machines cost at least $\$ 1.2$ million.

Even though the price of the PET/MRI scanner has yet to be determined, it can be inferred from the prices of other diagnostic imaging machines that the cost will be a few million dollars. It is predicted that a PET/MRI scanner will cost more than a PET/CT scanner did when it was the latest hybrid machine. Of course, this estimate is based on current trends in the industry and is subject to variability and issues of reimbursement as well as future market trends.

\section{Clinical Considerations}

${ }^{18}$ F-FDG PET has already had a huge valuable outcome on cancer treatment, and its use in clinical oncology practice continues to develop $(39,40)$. PET/CT is currently used mainly for whole-body oncologic evaluation, an application comprising most of the reimbursable indications for PET/CT (41). A PET/CT scan gives radiation therapists a more accurate anatomic reference point for intensitymodulated radiotherapy procedures, telling them the functional size and shape of a tumor and showing them precisely where to target the beam of radiation. This knowledge ensures that the tumor receives the maximum amount of radiation while the healthy tissue surrounding the tumor is spared (42).

To replace PET/CT, PET/MRI should be able to provide a better alternative, particularly in whole-body imaging.

Is PET/CT unanimously recognized as the standard imaging technology for clinical oncology? One should bear in mind that this issue is still controversial, since many investigators claim that PET/CT has a limited role in many indications, including lymphomas, lung nodules, and brain tumors (28). Time will dictate whether PET/MRI will influence the standard for future PET instrumentation, which is poised to advance molecular imaging and influence clinical and research practice.

\section{Reimbursement Issues}

Reimbursement issues are driven mainly by prospective clinical studies that demonstrate improvements in health outcomes conveyed by an imaging modality for a given indication. Therefore, given the higher soft-tissue contrast resolution of MRI and the fact that it has the highest sensitivity and specificity for many indications (e.g., detection of liver metastases) (31), coverage for PET scans is expected to be expanded.

\section{PRACTICE CONSIDERATIONS}

In practice, running a PET/CT scanner in a clinical environment to the uppermost diagnostic standards is not straightforward. Translating the experience and know-how gained in radiology to a nuclear medicine department and vice versa is not that easy because of the controversies surrounding PET/CT and the existing territorial and protective practices in health care facilities. Careful patient preparation and positioning are key elements of the long chain of data acquisition and processing protocols and require extensive training of the technologists operating the scanner to minimize artifacts and reduce interpretative pitfalls.

Licensure laws for imaging technologists vary from state to state. Currently, 38 states fully or partially license radiographers and 21 states fully or partially license nuclear medicine technologists (43). Licensure is designed to protect the public by ensuring that only qualified individuals engage in a given occupation or profession. State licensure of radiologic technologists and nuclear medicine technologists ensures that these individuals possess a basic level of education, knowledge, and skill. However, many of the state licensure laws are not prepared to deal with personnel who use dual ionizing radiation (SPECT/CT and PET/CT) equipment. For example, some states require that people operating PET/CT equipment possess dual certification in nuclear medicine and radiography - a very rare type of individual in some areas of the country. If a dually certified individual is not available, then these states require that 2 technologists be present to operate the PET/CT equipment-one who is licensed in nuclear medicine to perform the PET portion of the examination and one who is licensed in radiography to perform the CT portion of the examination. Requiring that technologists possess dual certification or that 2 technologists be present for PET/CT examinations could limit patient access to such valuable hybrid technology (44). Combining PET with MRI may be a natural answer to this dilemma.

\section{CONCLUSION}

It is interesting to look at the history of NMR and its origins within nuclear medicine technology and how it now may come around full circle back into the field. This overview of PET/MRI technology has discussed the history of NMR and the development of MRI today, outlined the differences between PET/CT and PET/MRI hybrid technology, and discussed some unique characteristics of PET/ MRI that may lead to its being the technology of choice in the future. Over the next 5 to 10 years, it will be interesting to see whether PET/MRI will replace PET/CT or whether PET/MRI will fade away as just another imaging technology that had potential but was never totally embraced clinically. PET/MRI appears to have more pros than cons and could become the melded modality of choice in the future, but time will tell the fate of this technology.

\section{REFERENCES}

1. Catana C, Procissi $\mathrm{D}, \mathrm{Wu} \mathrm{Y}$, et al. Simultaneous in vivo positron emission tomography and magnetic resonance imaging. Proc Natl Acad Sci USA. 2008;105:3705-3510.

2. Zaidi H, Mawlawi O. Simultaneous PET/MR will replace PET/CT as the molecular multimodality imaging platform of choice. Med Phys. 2007;34:15251528 .

3. Pichler BJ, Judenhofer MS, Pfannenberg C. Multimodal imaging approaches: PET/CT and PET/MRI. Handb Exp Pharmacol. 2008;(185 Pt 1):109-132.

4. Taschereau R, Chow PL, Chatziioannou AF. Monte Carlo simulations of dose from microCT imaging procedures in a realistic mouse phantom. Med Phys. 2006;33:216-224. 
5. Pietrzyk U. Recent aspects of hybrid imaging. Lecture presented at: Centre de Physique des Particules de Marseille; October 27-28, 2008, Marseille, France.

6. Gadian DG. NMR and Its Applications to Living Systems. New York, NY: Oxford University Press; 1995.

7. Toga AW, Mazziotta JC, eds. Brain Mapping: The Methods. 2nd ed. San Diego, CA: Academic; 2002.

8. Bulte JWM, Kraitchman DL. Monitoring cell therapy using iron oxide MR contrast agents. Curr Pharm Biotechnol. 2004;5:567-584.

9. Gustafsson B, Youens S, Louie AY. Development of contrast agents targeted to macrophage scavenger receptors for MRI of vascular inflammation. Bioconjug Chem. 2006;17:538-547.

10. Modo M, Cash D, Mellodew K, et al. Tracking transplanted stem cell migration using bifunctional, contrast agent-enhanced, magnetic resonance imaging. Neuroimage. 2002;17:803-811.

11. Perez JM, Josephson L, O'Loughlin T, Högemann D, Weissleder R. Magnetic relaxation switches capable of sensing molecular interactions. Nat Biotechnol. 2002;20:816-820.

12. Marzola P, Osculati F, Sbarbati A. High field MRI in preclinical research. Eur J Radiol. 2003;48:165-170.

13. Nelson SJ, Vigneron DB, Star-Lack J, Kurhanewicz J. High spatial resolution and speed in MRSI. NMR Biomed. 1997;10:411-422.

14. Morris PG. Magnetic resonance imaging and magnetic resonance spectroscopy assessment of brain function in experimental animals and man. $J$ Psychopharmacol. 1999;13:330-336.

15. Pathak AP, Gimi B, Glunde K, Ackerstaff E, Artemov D, Bhujwalla ZM. Molecular and functional imaging of cancer: advances in MRI and MRS. Methods Enzymol. 2004;386:3-60.

16. Pettegrew JW, Klunk WE, Panchalingam K, McClure RJ, Stanley JA. Molecular insights into neurodevelopmental and neurodegenerative diseases. Brain Res Bull. 2000;53:455-469.

17. Ross B, Bluml S. Magnetic resonance spectroscopy of the human brain. Anat Rec. 2001;265:54-84.

18. Golay X, Jiang H, van Zijl PCM, Mori S. High-resolution isotropic 3D diffusion tensor imaging of the human brain. Magn Reson Med. 2002;47:837843.

19. Pajevic S, Aldroubi A, Basser PJ. A continuous tensor field approximation of discrete DT-MRI data for extracting microstructural and architectural features of tissue. J Magn Reson. 2002;154:85-100.

20. Dafni H, Landsman L, Schechter B, Kohen F, Neeman M. MRI and fluorescence microscopy of the acute vascular response to VEGF165: vasodilation, hyperpermeability and lymphatic uptake, followed by rapid inactivation of the growth factor. NMR Biomed. 2002;15:120-131.

21. Howseman AM, Bowtell RW. Functional magnetic resonance imaging: imaging techniques and contrast mechanisms. Philos Trans R Soc London Ser B. 1999; 354:1179-1194.

22. Kim SG, Ugurbil K. High-resolution functional magnetic resonance imaging of the animal brain. Methods. 2003;30:28-41.

23. Logothetis NK, Guggenberger H, Peled S, Pauls J. Functional imaging of the monkey brain. Nat Neurosci. 1999;2:555-562.
24. Bombardieri E, Buscombe J, Lucignani G, Schober O. Advances in Nuclear Oncology: Diagnosis and Therapy. London, U.K.: Informa Healthcare; 2007.

25. Burbar Z, Grazioso RF, Corbeil J, et al. PET performance of MR/PET brain insert tomograph. In: Proceedings of the IEEE Nuclear Science Symposium and Medical Imaging Conference. Piscataway, NJ: IEEE; 2006.

26. Hill DLG, Batchelor PG, Holden M, Hawkes DJ. Medical image registration. Phys Med Biol. 2001;46:R1-R45.

27. Woods RP, Mazziotta JC, Cherry SR. MRI-PET registration with automated algorithm. J Comput Assist Tomogr. 1993;17:536-546.

28. Alavi A, Mavi A, Basu S, et al. Is PET-CT the only option? Eur J Nucl Med Mol Imaging. 2007;34:819-821.

29. Mankoff DA, Muzi M, Zaidi H. Quantitative analysis in nuclear oncologic imaging. In: Zaidi H, ed. Quantitative Analysis of Nuclear Medicine Images. New York, NY: Springer; 2006:494-536.

30. Cohade C, Wahl RL. Applications of positron emission tomography/computed tomography image fusion in clinical positron emission tomography: clinical use, interpretation methods, diagnostic improvements. Semin Nucl Med. 2003; 33:228-237.

31. Seemann MD. Whole-body PET/MRI: the future in oncological imaging. Technol Cancer Res Treat. 2005;4:577-582.

32. Marsden PK, Strul D, Keevil SF, Williams SC, Cash D. Simultaneous PET and NMR. Br J Radiol. 2002;75(suppl):S53-S59.

33. Catana C, Wu Y, Judenhofer MS, Qi J, Pichler BJ, Cherry SR. Simultaneous acquisition of multislice PET and MR images: initial results with a MRcompatible PET scanner. J Nucl Med. 2006;47:1968-1976.

34. Zaidi H, Montandon M-L, Slosman DO. Magnetic resonance imaging-guided attenuation and scatter corrections in three-dimensional brain positron emission tomography. Med Phys. 2003;30:937-948.

35. Zaidi $H$. Is MR-guided attenuation correction a viable option for dual-modality PET/MR imaging? Radiology. 2007;244:639-642.

36. Bolus NE. Radiation injury from diagnostic and therapeutic sources [abstract]. J Nucl Med. 2005;46(suppl):253P.

37. Cherry SR. Multimodality in vivo imaging systems: twice the power or double the trouble? Annu Rev Biomed Eng. 2006;8:35-62.

38. Pichler BJ, Judenhofer MS, Catana C, et al. Performance test of an LSO-APD detector in a 7-T MRI scanner for simultaneous PET/MRI. J Nucl Med. 2006; 47:639-647.

39. Czernin J, Allen-Auerbach M, Schelbert HR. Improvements in cancer staging with PET/CT: literature-based evidence as of September 2006. J Nucl Med. 2007;48 (suppl 1):78S-88S.

40. Blodgett TM, Meltzer CC, Townsend DW. PET/CT: form and function. Radiology. 2007;242:360-385.

41. Beebe M, Dalton J, Espronceda M, Evans D, Glenn R. CPT 2007: Professional Edition. Chicago, IL: American Medical Association Press; 2007.

42. Clarke M. Fusion imaging: a new type of technologist for a new type of technology. Statement presented at: PET-CT Consensus Conference; July 31, 2002; New Orleans, LA.

43. Licensing: Medical Imaging and Radiation Therapy-USA. Available at: http:// www.medhunters.com/articles/licensingMedicalImagingUsa.html. Accessed April 23, 2009 . 